\title{
A Matched Case-Control Study Measuring the Effectiveness of the Rotavirus Vaccines to Prevent Gastroenteritis Hospitalizations
}

\author{
Francisco Gimenez-Sanchez ${ }^{1 *}$, Elena Cobos-Carrascosa ${ }^{1}$, Miguel Sanchez-Forte ${ }^{1}$, Yolanda Gonzalez-Jimenez ${ }^{1}$, Ernestina Azor-Martine ${ }^{1}$ and Pablo Garrido- \\ Fernandez ${ }^{2}$ \\ ${ }^{1}$ Pediatrics Department, Hospital Torrecardenas, Almeria, Spain \\ ${ }^{2}$ FIBAO, Hospital Torrecardenas, Almeria, Spain \\ "Corresponding author: Francisco Gimenez-Sanchez, Pediatrics Department, Hospital Torrecardenas, Almeria, Spain, Tel: 34950206573; E-mail: \\ franciscogimenezsanchez@gmail.com
}

Received date: 18 January 2014; Accepted date: 11 February 2015; Published date: 16 February 2015

Copyright: (C) 2015 Gimenez-Sanchez F, et al. This is an open-access article distributed under the terms of the Creative Commons Attribution License, which permits unrestricted use, distribution, and reproduction in any medium, provided the original author and source are credited.

\begin{abstract}
Rotavirus has been identified as an important cause of diarrhea hospitalizations. Two oral live rotavirus vaccines have been licensed in Europe since 2006 and have been available in the Spanish market since then. The main objective of this study was to estimate the effectiveness of the rotavirus vaccination in the prevention of admissions caused by rotavirus infections using a matched case-control study in a hospital setting.
\end{abstract}

Patients and methods: Prospective, hospital based, matched case-control study including patients between aged between 2 months and 5 years, hospitalized during 2008-2010 with a diagnosis of Rotavirus Acute Gastroenteritis (RV+AGE) matched against two patients diagnosed as Rotavirus negative Acute Gastroenteritis (Control group A) and five patients hospitalized with non-gastrointestinal conditions (Control group B) (i.e. in the ratio $1: 2: 5)$.

Results: 466 patients were included: 57 cases of RV+AGE, 104 patients in Control group A and 305 in Control group B. No differences were found among the 3 groups when comparing demographic data. Vaccine effectiveness was estimated as $86 \%(95 \% \mathrm{Cl} 59-95)$ compared to group A and $88 \%(95 \% \mathrm{Cl} 68-95)$ compared to group B. Prior rotavirus vaccination was more frequent in mild cases than in those with moderate-severe symptoms and the length of admission was shorter in vaccine recipients (1.7 \pm 0.8 days) compared to those non-vaccinated (3.2 \pm 1.4 days) $(p<0.001 ; 95 \% \mathrm{Cl}: 1.3-1.7)$.

Conclusions: Our study found the rotavirus vaccines to be highly effective in preventing rotavirus-related hospitalizations and to reduce the severity of the rotavirus disease.

Keywords Rotavirus vaccines; Gastroenteritis; Preventing rotavirus; Diarrhea

\section{Introduction}

Rotavirus is the most common cause of severe acute gastroenteritis in infants and young children worldwide [1]. In Europe, several studies have identified Rotavirus as an important cause of diarrhea hospitalizations producing a vast number of admissions, especially in winter months, coinciding with the influenza and Respiratory Syncytial Virus seasons. The greatest disease burden is found in children aged $<2$ years [2-4]. In Spain, rotavirus has been estimated to account yearly for approximately 181,626 Rotavirus Acute Gastroenteritis (AGE) episodes among children, including 14,342 hospitalizations, 41,701 emergency room visits, and 48,320 outpatient visits [5]. The annual costs of Rotavirus AGE in children $<5$ years of age have been estimated at $€ 28.6$ million from the National Health System perspective while, from the societal perspective, they were estimated at $€ 50.0$ million.

The World Health Organization (WHO) has recommended the inclusion of rotavirus vaccines into all national immunization programmes [6,7]. Two oral live rotavirus vaccines, Rotarix (human monovalent vaccine containing genotype G1P [8] developed by GlaxoSmithKline, Brentford, United Kingdom) and RotaTeq (humanbovine pentavalent vaccine containing G1-4 and P [8] developed by Merck, Whitehouse Station, NJ) have been licensed in Europe since 2006 and have been available in the Spanish market since then. However, concerns regarding the laboratory finding of fragments of a circovirus in rotavirus vaccines lead the Spanish Agency of Medicines to issue a ban on these in 2010 that still stands for Rotarix, despite the fact that no adverse events of clinical importance in humans have been identified in association with these findings, and that both vaccines have demonstrated good safety and efficacy profiles in large clinical trials, in accordance with WHO, EMA (European Medicines Agency) and FDA (Food and Drug Administration) recommendations [7].

A significant reduction in the incidence of childhood diarrhea leading to hospitalization has been reported after the introduction of either routine childhood rotavirus vaccination or targeted vaccination recommendations in several countries, including Spain [8-10]. Despite the availability of rotavirus vaccines and convincing results from clinical trials and ecological studies, by 2013 only a small number of European countries had introduced routine rotavirus vaccination in the recommended childhood immunization schedule. In Spain, this policy has not been adopted for the public health service, but several 
Citation: Gimenez-Sanchez F, Cobos-Carrascosa E, Sanchez-Forte M, Gonzalez-Jimenez Y, Azor-Martine E, et al. (2015) A Matched CaseControl Study Measuring the Effectiveness of the Rotavirus Vaccines to Prevent Gastroenteritis Hospitalizations . J Vaccines Vaccin 6: 275. doi:10.4172/2157-7560.1000275

Page 2 of 4

medical societies recommend its administration, and it is implemented in the private market $[11,12]$. These facts lead to difficulties in the assessment of rotavirus vaccines uptake and of their effects on the population. Moreover, recent data suggest that rotavirus vaccine effectiveness may vary by geographic region [13]. In Europe, some differences in epidemiological characteristics of rotavirus infections have been reported [14]. Thus, studies monitoring rotavirus effectiveness post-marketing, under conditions of routine use, are essential to inform vaccination policies.

With rotavirus vaccines increasingly being introduced into childhood immunization programs, monitoring effectiveness in real life settings is a high priority and has been requested by the European Medicines Agency. The main objective of this study was to estimate the effectiveness of rotavirus vaccination in the prevention of admissions caused by rotavirus infection using a matched case-control study in a hospital setting.

\section{Patients and Methods}

Prospective, hospital based, matched case-control study including patients between aged between 2 months and 5 years, hospitalized during 2008-2010 with a diagnosis of Rotavirus AGE (RV+AGE). Every patient was matched against two patients diagnosed as Rotavirus negative AGE (RV-AGE) (Control group A) and five patients hospitalized with non-gastrointestinal conditions (Control group B) (i.e. in the ratio 1:2:5). The minimum sample size was calculated based on $90 \%$ power, 0.05 alpha value, a hypothetical vaccine effectiveness of $80 \%$, and an estimated $40 \%$ vaccine coverage (based on sales reported from IMS Health). With these parameters, the minimum sample size needed was 46 cases. All controls were matched by age and birth date (with a maximum of 8 weeks difference), gender, and same date of admission. Rotavirus cases were diagnosed in stool samples tested using a commercial enzyme immunoassay (EIA) collected within 3 days of admission. Demographic data, cohabiting siblings, clinical findings using the Ruuska-Vesikari scale (calculated with data available for the full duration of the episode of gastroenteritis) as previously described [15], Past medical history and vaccination schedule were analyzed. Immunization data were obtained from medical records. Parents were interviewed and asked to provide immunization information for those patients whose records did not include it. Rotavirus vaccination status was classified as positive with at least one dose administered. Institutional Review Board approval was obtained from the local Research Committee.

Data were analyzed by bivariant logistic regression using the computer programs SPSS version 17.0. Statistical significance was defined as $\mathrm{P}<0.05$, all $\mathrm{P}$ values were 2 -sided. Vaccine effectiveness was calculated as follows: $\mathrm{VE}=(1$-Odds Ratio $) \times 100$. Cases that had received their first rotavirus vaccination $<14$ days prior to the onset of rotavirus symptoms were excluded from the analysis. For controls, vaccine doses given $<14$ days before the date of their matched case's symptom onset were not included.

To estimate the coverage of the rotavirus vaccines, sales in the hospital's area of influence were obtained from IMS Health. The expected number of vaccinees was obtained by dividing the total doses by 2 or 3 doses depending on the vaccine used. Coverage was then estimated by dividing the estimated number of vaccinees by the registered number of children under 1 year of age born in that same period in the catchment area of our hospital.

\section{Results}

During the study period, a total of 466 patients were included: 57 cases of RV+AGE, 104 patients in control group A (RV-AGE) and 305 in Control group $\mathrm{B}$. The average age for all the cases and controls were $13.07 \pm 9.62$ months and $59.2 \%$ were males. No differences were found among the 3 groups when comparing age, sex, prematurity (gestational age $<37$ weeks), breastfeeding, cohabiting siblings, and country of origin of parents, (Table 1). Most cases of RV+AGE were found in 2008 (56\%) compared to 2009 (23\%) and 2010 (21\%) $(\mathrm{p}<0.001)$. RV-AGE cases were equally distributed among 2008 (33\%), 2009 (38\%) and 2010 (29\%). On analysing the severity of AGE in cases and A group controls, RV+AGE cases were more likely to be classified as moderate or severe (69\% among cases compared to $38 \%$ in group A controls $(\mathrm{p}<0.001 ; \mathrm{OR}=3.57$; IC 95\%,1.82-7.04).

\begin{tabular}{|l|l|l|l|l|l|}
\hline \multirow{2}{*}{$\begin{array}{l}\text { Social and } \\
\text { demographic } \\
\text { characteristi } \\
\text { cs }\end{array}$} & $\begin{array}{l}\text { Cases } \\
\mathbf{( 5 7 )}\end{array}$ & \multicolumn{2}{|l|}{ Control A Group (-104) } & \multicolumn{2}{l}{ Control B Group (305) } \\
\cline { 2 - 6 } & $\mathbf{N}(\%)$ & $\mathbf{N}(\%)$ & $\mathbf{p}$ & $\mathbf{N}(\%)$ & $\mathbf{P}$ \\
\hline $\begin{array}{l}\text { Age (months) } \\
\text { (Median } \\
\text { SD) }\end{array}$ & $10 \pm 6.30$ & $15 \pm 10.18$ & 0.12 & $12 \pm 9.78$ & 0.36 \\
\hline Sex (male) & $35(61.4 \%)$ & $69(66.3 \%)$ & 0.53 & $172(56.4 \%)$ & 0.66 \\
\hline Prematurity & $4(7.0 \%)$ & $15(14.4 \%)$ & 0.16 & $41(13.4 \%)$ & 0.17 \\
\hline Breastfeeding & $29(50.9 \%)$ & $46(44.2 \%)$ & 0.41 & $119(39.0 \%)$ & 0.09 \\
\hline Siblings & $28(49.1 \%)$ & $45(43.3 \%)$ & 0.47 & $139(45.6 \%)$ & 0.62 \\
\hline Country of origin & $48(84.2 \%)$ & $85(81.8 \%)$ & - & $273(89.5 \%)$ & - \\
\hline Spanish & $9(15.8 \%)$ & $19(18.2 \%)$ & 0.69 & $32(10.5 \%)$ & 0.25 \\
\hline Foreign & 9 & & & & \\
\hline
\end{tabular}

Table 1: Characteristics of the subjects.

Regarding vaccination status, five patients $(8 \%)$ had received 1 or more doses of a rotavirus vaccine in the case group, forty-one (40\%) in Control group A and $132(43 \%)$ in Control group B. Vaccine effectiveness was estimated as $86 \%(95 \% \mathrm{CI}$ 59-95) compared to Control group A and 88\% (95\%CI 68-95) compared to Control group B. (Figure 1).

\section{Discussion}

The effectiveness of rotavirus vaccines has been reported in numerous studies based on hospitals, emergencies departments and primary care settings, or using population-based surveillance methodology [16,17]. Our report represents the first case-control study matched by gender and age that analyses risk factors for rotavirus infection conducted in Spain, and contributes to assessing rotavirus vaccine effectiveness in Europe. Recently, several casecontrol studies carried out in Europe have been published, but few of them were matched by sex, age and timeframe of admission [18-21]. In our study, this matching has improved the comparability among the three groups with regard to risk factors for rotavirus disease. The casecontrol design we used, including two different matched control groups (non-rotavirus AGE and non-AGE hospitalized patients) provided comparison groups that were demographically similar to the matched cases, enhancing comparability. Since Control group B did not have AGE misclassification of cases and these controls was 
Citation: Gimenez-Sanchez F, Cobos-Carrascosa E, Sanchez-Forte M, Gonzalez-Jimenez Y, Azor-Martine E, et al. (2015) A Matched CaseControl Study Measuring the Effectiveness of the Rotavirus Vaccines to Prevent Gastroenteritis Hospitalizations . J Vaccines Vaccin 6: 275. doi:10.4172/2157-7560.1000275

Page 3 of 4

unlikely. Other non-matched case-control studies that have investigated rotavirus vaccine effectiveness in Spain have been published recently [19-21]. In those studies, controls were older, less frequently hospitalized, and were seen in a different timeframe, making it necessary to use adjusted multiple logistic regression analyses. These factors have been incorporated in our matched study. Another issue to highlight is the high percentage of patients who had received at least one dose of vaccine among the control groups $(40 \%$ and $43 \%$ respectively), compared with the low coverage found in other studies in Spain. Rotavirus vaccine uptake in Spain has been estimated using sales by region and this shows wide differences between them ranging from $6 \%$ to $63 \%$ (data unpublished, IMS Health). Such variance could be influenced by social and economic factors together with the knowledge, attitudes and practices of healthcare providers.

$\%$ subjects vaccinated $(n)$

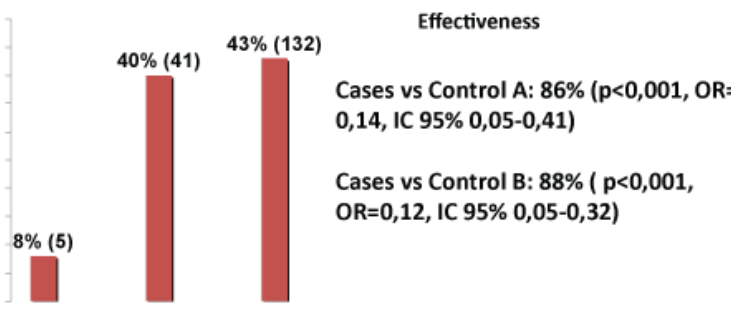

Figure 1: Vaccine Effectiveness in the prevention of hospitalizations caused by Rotavirus AGE.

Prior rotavirus vaccination was more frequent in mild cases than in those with moderate-severe symptoms (Figure 2). In addition, the average length of admission was shorter in vaccine recipients $(1.7 \pm 0.8$ days) compared to those who had not been vaccinated ( $3.2 \pm 1.4$ days) $(\mathrm{p}<0.001 ; 95 \%$ CI: 1.3-1.7)

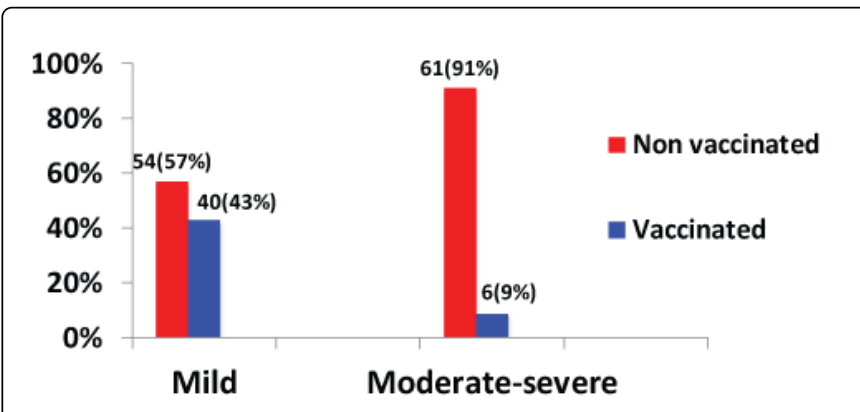

Figure 2: Antecedent of Rotavirus vaccination related to severity (cases and control group A pooled).

Our vaccine effectiveness estimates are similar to results found in other postmarketing case-control studies, showing a slightly lower effectiveness when compared with the results from randomized controlled trials [22-24]. However, Castilla et al. [20] and BellidoBlasco et al (21) also using a case-control design in Spain, reported a vaccine effectiveness of $83 \%$ and $88 \%$ for hospitalized rotavirus cases among children aged $<5$ years and $<3$ years, respectively. This data is virtually identical to ours, although the study was from a different geographic region with a much lower vaccine uptake. Another study conducted in Spain, including pooled patients who were hospitalized attended to in emergency departments and in the primary care system, with similar a rotavirus vaccine coverage to ours, found a vaccine effectiveness of $95 \%$ for the prevention of hospitalizations and $92 \%$ for any episode of AGE caused by rotavirus [19]. Additionally, these studies did not find differences in the effectiveness of the two brands of rotavirus vaccine marketed. With a similar methodology, another study in Israel showed a vaccine effectiveness of $89 \%$ using nonrotavirus AGE as controls [25]. Most studies conducted so far have found a lower level of protection when analysing cases of AGE in outpatient care, and a higher effectiveness in the prevention of cases of severe disease $[26,27]$. In our study, an important factor to take into account is that the Rate Ratios were analysed in hospitalized children and were not combined with outpatient cases, a fact that could give a better assessment of the risk of rotavirus infection. Recently, another matched case-control study, conducted in Belgium during the same time period as ours, found $90 \%$ vaccine effectiveness against rotavirus admissions. They considered children as vaccinated if they had received two doses of the monovalent rotavirus vaccine [18]. Data from developing countries with rotavirus vaccines have reported high effectiveness. A study in Nicaragua showed $83 \%$ vaccine effectiveness among children younger than 12 months, using a pentavalent vaccine, and a study from Brazil found a $76 \%$ using a monovalent vaccine [28-29]. Both these studies mentioned were matched case-control studies, and reported a lower effectiveness when cases were compared to hospitalized controls rather than those who were not hospitalized.

Our study found that rotavirus infected patients who had been vaccinated had a milder clinical presentation than the unvaccinated, which was similar to findings from another case control study conducted in Germany [30]. This could be explained by the likely capacity of rotavirus vaccines to confer partial protection, leading to less severe cases. Moreover, vaccinated subjects had a shorter length of admission compared with the unvaccinated, suggesting a faster recovery rate among them. It has been shown that the complete course of rotavirus vaccinations leads to a higher effectiveness compared to that among partially vaccinated children [19], but in our study the number of rotavirus vaccine administrated was not measured so this could not be correlated with the severity of disease, which could be considered a limitation of the study.

Our study has other limitations: the vaccine type was not analysed because this was not normally recorded in the immunization records by nurses. Thus, possible differences in effectiveness between the monovalent and pentavalent vaccines could not be measured. A potential bias could have been introduced because AGE cases might be misclassified if there are issues with the reliability of the rotavirus EIA results that could have led to a bias towards the null hypothesis. In our study, such misclassification should not have had a major impacted on our results, since the rotavirus EIA is reported to be $>95 \%$ sensitive [31] and more than $95 \%$ of bulk stool specimens were collected within the first 48 hours. However, stool samples were not stored to be typed by molecular methods in future studies, which could be considered another shortcoming of the study. Finally, the number of rotavirus vaccine doses was not recorded so vaccine effectiveness based on the number of doses received could not be calculated. Thus, a bias towards the null (lower vaccine effectiveness) could have derived from the misclassification of children vaccinated with just one dose among those vaccinated with more than one dose.

In conclusion, our study found rotavirus vaccines to be highly effective in preventing rotavirus-related hospitalizations. The 
Citation: Gimenez-Sanchez F, Cobos-Carrascosa E, Sanchez-Forte M, Gonzalez-Jimenez Y, Azor-Martine E, et al. (2015) A Matched CaseControl Study Measuring the Effectiveness of the Rotavirus Vaccines to Prevent Gastroenteritis Hospitalizations . J Vaccines Vaccin 6: 275. doi:10.4172/2157-7560.1000275

Page 4 of 4

effectiveness we found was similar to that obtained in studies from other European regions some of which used different methodologies. In addition, a reduction in the length of hospital stays due to rotavirus infection was observed among vaccinated cases, suggesting effectiveness also against the disease's severity. Our results should be useful to establish future recommendations for rotavirus vaccination.

\section{References}

1. Tate JE, Patel MM, Steele AD, Gentsch JR, Payne DC, et al. (2010) Global impact of rotavirus vaccines. Expert Rev Vaccines 9: 395-407.

2. Diez-Domingo J, Baldo JM, Patrzalek M, Pazdiora P, Forster J, et al (2001) Primary care-based surveillance to estimate the burden of rotavirus gastroenteritis among children aged less than 5 years in six European countries. Eur J Pediatr 170: 213-222.

3. Giaquinto C, van Damme P, REVEAL Study Group (2010) Age distribution of paediatric rotavirus gastroenteritis cases in Europe: the REVEAL study. Scand J Infect Dis 42: 142-147.

4. Gimenez-Sanchez F, Delgado-Rubio A, Martinon-Torres F, BernaolaIturbe E, Rotascore Research Group (2010) Multicenter prospective study analysing the role of rotavirus on acute gastroenteritis in Spain. Acta Paediatr 99: 738-742.

5. Diez-Domingo J, Surinach NL, Alcalde NM, Betegón L, Largeron N, et al. (2010) Burden of paediatric Rotavirus Gastroenteritis (RVGE) and potential benefits of a universal Rotavirus vaccination programme with a pentavalent vaccine in Spain. BMC public health 10: 469.

6. WHO (2013) Releve epidemiologique hebdomadaire/Section d'hygiene du Secretariat de la Societe des Nations. Weekly Epidem Rec 88: 49-64

7. Bouzón Alejandro M, Diez Domingo J, Martinón-Torres F (2011) Circovirus and impact of temporary withdrawal of rotavirus vaccines in Spain. Hum Vaccin 7: 798-799.

8. Hemming $M$, Räsänen $S$, Huhti L, Paloniemi M, Salminen $M$, et al (2013) Major reduction of rotavirus, but not norovirus, gastroenteritis in children seen in hospital after the introduction of RotaTeq vaccine into the National Immunization Programme in Finland. Eur J Pediatr 172: 739-746.

9. Desai R, Curns AT, Steiner CA, Tate JE, Patel MM, et al. (2012) All-cause gastroenteritis and rotavirus-coded hospitalizations among US children, 2000-2009. Clin Infect Dis 55: e28-34.

10. Hyde TB, Dentz H, Wang SA, Burchett HE, Mounier-Jack S, et al. (2012) The impact of new vaccine introduction on immunization and health systems: a review of the published literature. Vaccine 30: 6347-6358.

11. Giménez Sánchez F, Martinón Torres F, Bernaola Iturbe E, Baca Cots M, de Juan Martín F, et al. (2006) [The role of the rotavirus vaccine in childhood vaccination schedules]. An Pediatr (Barc) 64: 573-577.

12. Bernaola Iturbe E, Gimenez Sanchez F, Baca Cots M, de Juan Martin F, Diez Domingo J, et al. (2008) [Immunization schedule of the Spanish Association of Pediatrics: recommendations 2008]. An Pediatr (Barc) 68: 63-69.

13. Fischer Walker CL, Black RE (2011) Rotavirus vaccine and diarrhea mortality: quantifying regional variation in effect size. BMC Public Health 11 Suppl 3: S16.

14. Van Damme P, Giaquinto C, Maxwell M, Todd P, Van der Wielen M; REVEAL Study Group (2007) Distribution of rotavirus genotypes in Europe, 2004-2005: the REVEAL Study. J Infect Dis 195 Suppl 1: S17-25.

15. Ruuska T, Vesikari T (1990) Rotavirus disease in Finnish children: use of numerical scores for clinical severity of diarrhoeal episodes. Scand J Infect Dis 22: 259-267.
16. Donauer S, Payne DC, Edwards KM, Szilagyi PG, Hornung RW, et al. (2013) Determining the effectiveness of the pentavalent rotavirus vaccine against rotavirus hospitalizations and emergency department visits using two study designs. Vaccine 31: 2692-2697.

17. Desai SN, Esposito DB, Shapiro ED, Dennehy PH, Vázquez M (2010) Effectiveness of rotavirus vaccine in preventing hospitalization due to rotavirus gastroenteritis in young children in Connecticut, USA. Vaccine 28: 7501-7506.

18. Braeckman T, Van Herck K, Meyer N, Pircon JY, Soriano-Gabarro M, et al. (2012) Effectiveness of rotavirus vaccination in prevention of hospital admissions for rotavirus gastroenteritis among young children in Belgium: case-control study. Bmj 345: e4752.

19. Martinón-Torres F, Bouzón Alejandro M, Redondo Collazo L, Sánchez Lastres JM, Pértega Díaz S, et al. (2011) Effectiveness of rotavirus vaccination in Spain. Hum Vaccin 7: 757-761.

20. Castilla J, Beristain X, Martinez-Artola V, Navascues A, Garcia Cenoz M, et al.(2012) Effectiveness of rotavirus vaccines in preventing cases and hospitalizations due to rotavirus gastroenteritis in Navarre, Spain. Vaccine 30: 539-543.

21. Bellido-Blasco JB, Sabater-Vidal S, Salvador-Ribera Mdel M, ArnedoPena A, Tirado-Balaguer MD, et al. (2012) Rotavirus vaccination effectiveness: a case-case study in the EDICS project, Castellón (Spain). Vaccine 30: 7536-7540.

22. Boom JA, Tate JE, Sahni LC, Rench MA, Hull JJ, et al. (2010) Effectiveness of pentavalent rotavirus vaccine in a large urban population in the United States. Pediatrics 125: e199-207.

23. Vesikari T, Matson DO, Dennehy P, Van Damme P, Santosham M, et al. (2006) Safety and efficacy of a pentavalent human-bovine (WC3) reassortant rotavirus vaccine. N Engl J Med 354: 23-33.

24. Ruiz-Palacios GM, Perez-Schael I, Velazquez FR, Abate H, Breuer T, et al. (2006) Safety and efficacy of an attenuated vaccine against severe rotavirus gastroenteritis. The New England journal of medicine 354: 11-22.

25. Muhsen K, Shulman L, Kasem E, Rubinstein U, Shachter J, et al. (2010) Effectiveness of rotavirus vaccines for prevention of rotavirus gastroenteritis-associated hospitalizations in Israel: a case-control study. Hum vaccin 6:450-454.

26. Staat MA, Payne DC, Donauer S, Weinberg GA, Edwards KM, et al. (2011) Effectiveness of pentavalent rotavirus vaccine against severe disease. Pediatrics 128: e267-275.

27. Snelling TL, Andrews RM, Kirkwood CD, Culvenor S, Carapetis JR (2011) Case-control evaluation of the effectiveness of the G1P[8] human rotavirus vaccine during an outbreak of rotavirus $\mathrm{G} 2 \mathrm{P}[4]$ infection in central Australia. Clinical infectious 52: 191-199.

28. Mast TC, Khawaja S, Espinoza F, Paniagua M, Del Carmen LP, et al (2011) Case-control study of the effectiveness of vaccination with pentavalent rotavirus vaccine in Nicaragua. Pediatr Infect Dis J 30: e209-215.

29. Justino MC, Linhares AC, Lanzieri TM, Miranda Y, Mascarenhas JD, et al. (2011) Effectiveness of the monovalent G1P[8] human rotavirus vaccine against hospitalization for severe G2P[4] rotavirus gastroenteritis in Belem, Brazil. The Pediatric infectious disease journal 30: 396-401.

30. Adlhoch C, Hoehne M, Littmann M, Marques AM, Lerche A, et al (2013) Rotavirus vaccine effectiveness and case-control study on risk factors for breakthrough infections in Germany, 2010-2011. Pediatr infect dis j 32: e82-89.

31. Dennehy PH, Gauntlett DR, Spangenberger SE (1990) Choice of reference assay for the detection of rotavirus in fecal specimens: electron microscopy versus enzyme immunoassay. J clin microbiol 28:1280-1283. 